\title{
Radiation free-for-all is imminent
}

This editorial addresses a trend, that has become apparent over the past few years, concerning the highest healthcare professional body in the land: the Health Professions Council (HPCSA) of South Africa. A current process serves as a perfect example of how this body chooses to conduct its business. The methodology and potential outcome of the process need to be placed in the public arena, as the consequences concern all South Africans.

A unilateral process has been instituted by the HPCSA to embark on opening the diagnostic use of ionising radiation to all medical disciplines (specialist and non-specialist). This initiative has progressed to the point of being a fait accompli - but it has all happened without consultation and without the contribution of expert opinion in the field of radiation medicine.

International academic literature - especially over the past 5 years - has emphasised the established association between radiation exposure from computed tomography (a primary concern), as well as other sources of diagnostic X-rays, that leads to a significant increase in the risk for fatal cancer over a child's lifetime. The reviews aim to heighten awareness and spearhead efforts to reduce unnecessary radiation, and especially computed tomography (CT) scans, in children and young adults. The use of CT in these target age groups continues to grow; more than 60 million CT scans are estimated to be performed annually in the USA, of which 7 million are on children. ${ }^{1}$

Education and advocacy directed to referring clinicians reinforce these principles. Radiation exposure may be further reduced by developing clinical pathways that limit CT scanning and instead encourage alternative, non-radiation imaging modalities such as ultrasound and magnetic resonance imaging.

We view with alarm the actions of the HPCSA against the background of the growing concerns of an alerted world towards a radiation health issue that has two disconcerting components: (i) the startling increase in radiation-induced cancers in the young, and (ii) the effect and final outcome of accumulated dose on the human genetic pool. And so, while countries with a genuine concern for the effects of medical radiation are tightening the controls, we in South Africa have a council that seems recklessly intent on opening the radiation floodgates to all medical and medically-related fields (specialist and non-specialist). It is difficult to identify a rationale, as this country has an academically sound, morally strong and scientifically reliable programme for the use of ionising radiation in the diagnostic process. The specialty of Diagnostic Radiology, assisted by a Radiation Control Board and several departments of medical physics, as well as other experts in the field, stand central in this process. Properly trained specialists in imaging diagnosis, radiation protection and the process of weighing the potential hazards of ionising radiation against diagnostic gain, constantly ensure our international rating.

So what are the motives for wanting to destroy a well-established system in good working order? Some form of political expediency comes to mind. Why else do we witness decisions not based on scientific fact or academic foundation, without scientific survey or consultation with professional experts, and a refusal to grasp the consequences of a radiation carte blanche to all and sundry?

\section{Jan Lotz}

Editor-in-chief

1. ALARA: is there a cause for alarm? Reducing radiation risks from computed tomography scanning in children. Shah NB, Platt SL. Curr Opin Pediatr 2008; 20: 243-247. 